\title{
The astrometric data reduction software (ADRS) and error budget for PRIMA
}

\author{
N. M. Elias II $I^{1,2}$, R. N. Tubbs ${ }^{2}$, R. Köhler ${ }^{1,2}$, S. Reffert ${ }^{1}$, I. Stilz ${ }^{1,2}$, \\ R. Launhardt ${ }^{2}$, J. de Jong ${ }^{3}$, A. Quirrenbach ${ }^{1}$, F. Delplancke ${ }^{3}$, \\ Th. Henning ${ }^{2}$, D. Queloz ${ }^{4}$ and ESPRI Consortium ${ }^{1,2,3,4}$ \\ ${ }^{1}$ Zentrum für Astronomie (ZAH) der Universität Heidelberg \\ Landesternwarte (LSW); Köenigstuhl 12; 69117 Heidelberg, Germany \\ email: n.elias@lsw.uni-heidelberg.de \\ ${ }^{2}$ Max-Planck-Institut für Astronomie (MPIA) \\ Königstuhl 17; 69117 Heidelberg, Germany \\ ${ }^{3}$ European Southern Observatory (ESO) \\ Karl-Schwarzschild-Strasse 2; 85748 Garching bei München, Germany \\ ${ }^{4}$ Observatoire Astronomique de l'Université de Genève \\ 1290 Sauverny, Switzerland
}

\begin{abstract}
The Astrometric Data-Reduction Software (ADRS) processes fringe, delay, environmental, and calibration data for PRIMA narrow-angle astrometry. It is automated software designed to provide fully-calibrated differential delays and separation angles. The ADRS is divided into on-line and off-line processing. The former deals with calibration and data compression, while the latter applies corrections and calculates science quantities. PRIMA is the first VLTI instrument that may require removal of long-term environmental trends. The trend identification and fitting routines are not part of the distributed on-line and off-line processing software. Instead, files containing fit parameters will be updated regularly. Coding is presently underway. The PRIMA error budget summarizes the principal sources of error in PRIMA astrometric observations.
\end{abstract}

Keywords. astrometry, stars: planetary systems, instrumentation: interferometers, techniques: interferometric, methods: data analysis, techniques: high angular resolution, infrared: stars

\section{Introduction}

PRIMA will add a dual-beam capability to the VLTI, allowing both narrow-angle astrometry with the $1.8 \mathrm{~m}$ Auxiliary Telescopes (ATs) and the ability to do phase-referenced imaging (further details of PRIMA can be found in Launhardt et al. (2008)). The Astrometric Data-Reduction Software (ADRS) processes PRIMA fringe, delay, environmental, and calibration data for astrometric observations. It is not an interactive package and it does not fit planetary orbits. The primary purpose of the ADRS is to automatically produce calibrated differential delays and separation angles. It is based upon the PRIMA error budget (Tubbs et al. (2008)) and follows the standard interferometric paradigm for data reduction: averaging, calculation of intermediate quantities, and calibration.

The top-level ADRS requirements are: 1) the code must be as simple as possible to perform the job; 2) intermediate quantities should be calculated and saved in tables and header keywords, to assess data quality and verify the mathematical assumptions; and 3) the code must be expandable, to respond to new information, ideas, and algorithms. The same software must run at ESO/Paranal (responding to the PRIMA instrumentation) 


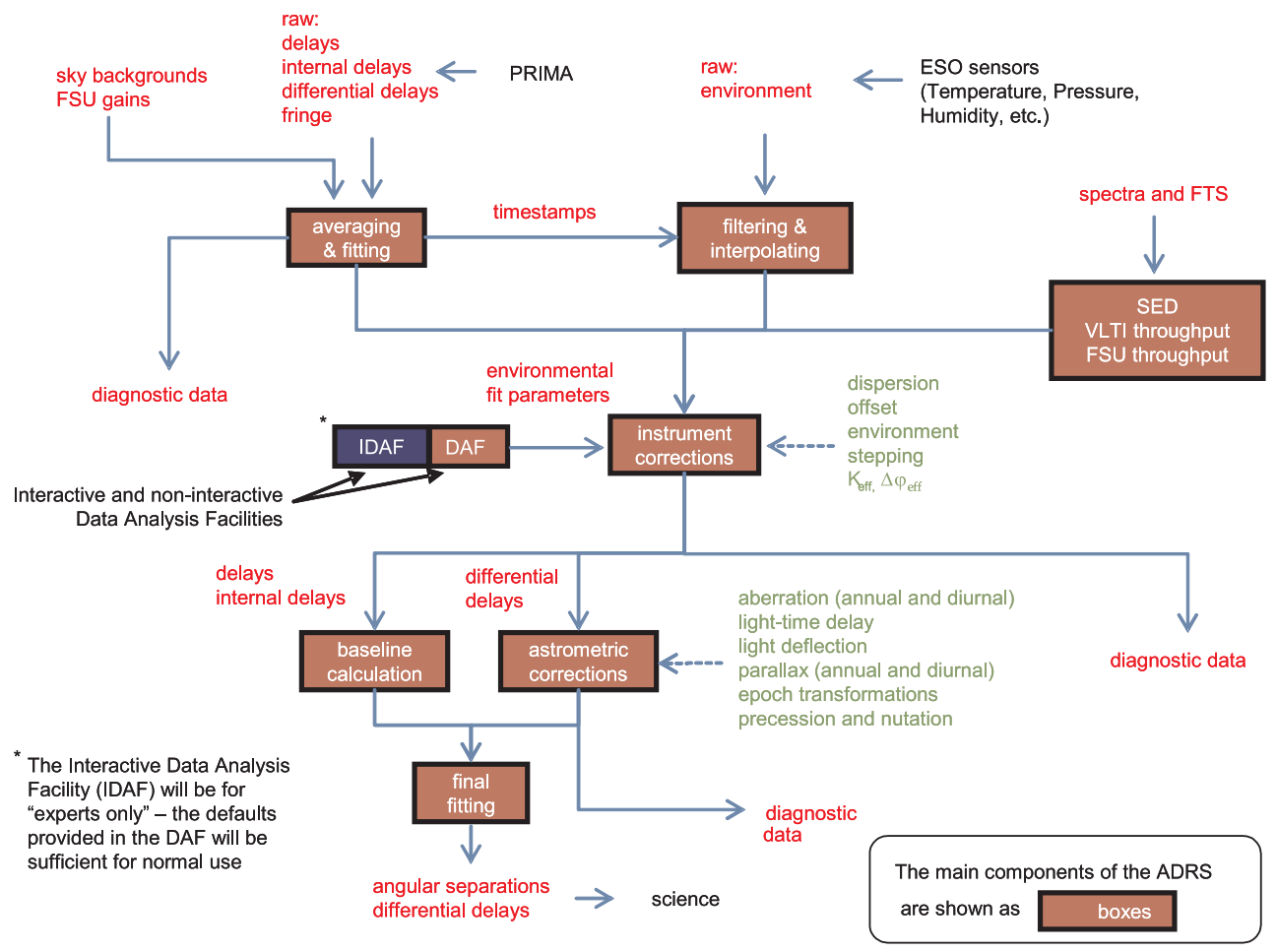

Figure 1. Flow diagram of the Astrometric Data-Reduction Software (ADRS)

and ESO/Garching (periodic reprocessing as fitted trends improve). Users can also run the ADRS, but we expect that the results produced by ESO will already be of the high quality required for scientific publications.

\section{Data flow}

The ADRS is divided into two main systems, on-line (pipeline) processing and offline processing. The output files are divided into data levels, with the highest data level corresponding to science-grade data.

The on-line processing is responsible for reducing calibration data, averaging fringe data, fitting delay data, and interpolating environmental data (top of Figure 1). To eliminate the need for additional interpolation, all fringe, delay, and environmental data are placed onto a fixed $\approx$ one-second time grid. A number of intermediate diagnostic quantities are also produced, such as total photon numbers, squared visibilities, and phase errors. On-line recipes include Fourier-transform spectroscopy, gain corrections, sky backgrounds, laboratory darks, and fringe/delay/environment data compression.

The off-line processing corrects delays and differential delays for environmental and astrometric effects (middle and bottom of Figure 1). The delays are corrected for disper- 
sion, and the differential delays are corrected for tracking errors and the arbitrary zero point. Environmental effects will likely consist of temperature, pressure, humidity, seeing, etc. Astrometric effects include light-time delay, light deflection, aberration, annual and diurnal parallax, and Earth orientation. The fringe data are used for dispersion and tracking corrections. The delay data are used to calculate the baseline vectors. The final data product consists of differential delays and star separations.

We expect long-term trends in delays and differential delays due to environmental effects. The PRIMA Consortium will use an IDL-based software package called the Interactive Data Analysis Facility (IDAF). It consists mostly of plotting and FITS-file routines, and it may eventually be expanded to include automated trend-identification routines.

After finding trends with the IDAF, the Data Analysis Facility (DAF) is used to select data and calculate fits that are used by the off-line processing recipes. The data selection part of the DAF is based upon the Organization, Classification, and Association (OCA) package. It is a rule-based interpreter that selects FITS files based on keywords in the primary headers. The fits, as well as instrument bandpasses and astrometric catalogs are saved in a group of files called the Correction Collection (CoCo). They are updated on a regular basis.

\section{Error budget}

The measurement errors in a single 30-minute PRIMA astrometric observation can be separated into errors in the knowledge of the narrow-angle baseline, and errors in the measurement of the differential delay using this baseline. The AT telescopes were designed before PRIMA, and two aspects of their design may limit the astrometric performance: the narrow-angle baseline stability and the differential delay introduced by the roughness of AT mirrors which lie close to an image plane. We are currently developing modeling and improvements for the ATs in order to overcome these problems. The PRIMA Error Budget is summarized in more detail in Tubbs et al. (2008).

\section{Conclusion}

The overall top-level design of the ADRS was finished in May 2007 at the Final Design Review (FDR). File formats have been created, but additional data may be saved as needed. Most recipes have been designed using work-breakdown structures, and preliminary versions of half of the recipes have already been written.

Additional functionality, which has already been planned, will be implemented in the near future. The beta versions will be delivered to ESO early in 2008. The commissioning versions will be delivered summer/fall 2008, where they will be debugged along with the hardware at the VLTI on Paranal.

Work on optimizing the operation of PRIMA and assessing the expected astrometric error is ongoing. 


\section{References}

Launhardt, R., Henning, Th., Queloz, D., Quirrenbach, A., Delplancke, F., Derie, F., Elias, N., Pepe, F., Reffert, S., Segransan, D., Setiawan, J., Tubbs, R., \& ESPRI Consortium 2008, The ESPRI project: Narrow-angle astrometry with VLTI-PRIMA (these proceedings).

Tubbs, R., Elias, N., Launhardt, R., Reffert, S., Delplancke, F., Quirrenbach, A. Henning, Th., Queloz, D., \& ESPRI Consortium 2008. ESPRI data-reduction strategy and error budget for PRIMA. In: Proc. IAUS 248 (in press). 